UCRL-JC-124858

PREPRINT

\title{
Wavefront Correction for Static and Dynamic Aberrations to within 1 Second of the System Shot in the NIF Beamlet Demonstration Facility
}

\author{
R. Hartley, M. Kartz, W. Behrendt, A. Hines, \\ G. Pollock, E. Bliss, T. Salmon, S. Winters, \\ B. Van Wonterghem, and R. Zacharias
}

This paper was prepared for submittal to the 2nd Annual International Conference on Solid-State Lasers for Application to ICF

Paris, France

October 22-25, 1996

October 21, 1996

This is a preprint of a paper intended for publication in a journal or proceedinge. Since changes may be made before publication, this preprint is made available with the underotanding that it will not be cited or reproduced without the permission of the author. 


\section{DISCLAIMER}

This document was prepared as an account of work sponsored by an agency of the United States Government. Neither the United States Government nor the University of California nor any of their employees, makes any warranty, express or implied, or assumes any legal liability or responsibility for the accuracy, completeness, or usefulness of any information, apparatus, product, or process

disclosed, or represents that its use would not infringe privately owned rights. Reference herein to any specific commercial product, process, or service by trade name, trademark, manufacturer, or otherwise, does not necessarily constitute or imply its endorsement, recommendation, or favoring by the United States Government or the University of California. The views and opinions of authors expressed herein do not necessarily state or reflect those of the United States Government or the University of California, and shall not be used for advertising or product endorsement purposes. 


\title{
Wavefront correction for static and dynamic aberrations to within 1 second of the system shot in the NIF Beamlet demonstration facility
}

\author{
Robert Hartley, Michael Kartz, William Behrendt, Andy Hines, George Pollock, \\ Erlan Bliss, Thad Salmon, Scott Winters, Bruno Van Wonterghem, Richard Zacharias
}

\author{
Lawrence Livermore National Laboratory, P.0. Box 808, \\ Livermore, CA 94550
}

\begin{abstract}
The laser wavefront of the NIF Beamlet demonstration system is corrected for static aberrations with a wavefront control system. The system operates closed loop with a probe beam prior to a shot and has a loop bandwidth of about $3 \mathrm{~Hz}$. However, until recently the wavefront control system was disabled several minutes prior to the shot to allow time to manually reconfigure its attenuators and probe beam insertion mechanism to shot mode.

Thermally-induced dynamic variations in gas density in the Beamlet main beam line produce significant wavefront error. Figure 1 shows the Beamlet peak-to-valley wavefront aberration as a function of time after the Beamlet wavefront controller is disabled. After about 5-8 seconds, the wavefront error has increased to a new, higher level due to turbulenceinduced aberrations no longer being corrected. This implies that there is a turbulence-induced aberration noise bandwidth of less than one Hertz, and that the wavefront controller could correct for the majority of turbulence-induced aberration (about one-third wave) by automating its reconfiguration to occur within one second of the shot. This modification was recently implemented on Beamlet; we call this modification the $t_{0}-1$ system.
\end{abstract}

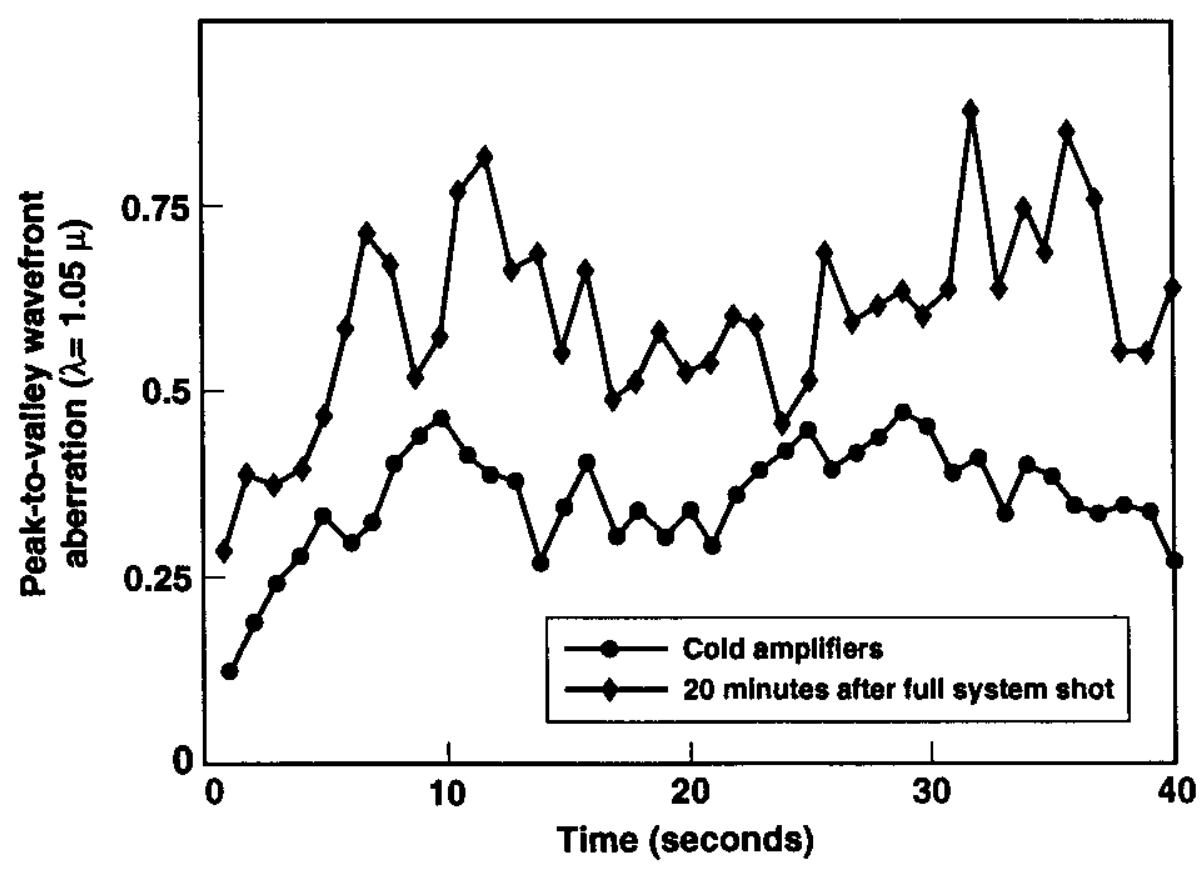

70-50-1096-2448pb01

Figure 1. Output wavefront evolution after interrupting closed loop deformable mirror control. 


\section{OVERVIEW}

Static and dynamic system aberrations, as shown in Figure 2, degrade the quality of the propagated wave front. Static aberrations include opticals figure errors and small scale errors inherent from material and machine tool operations. Dynamic sources of turbulence include thermal buoyancy or turbulence in the system as well as pump-induced amplifier thermal aberrations. Prior to implementation of the $t_{0}-1$ modifications, the adaptive optics system had the capability to precorrect for pump-induced aberrations but not for gas motion effects, as seen in Figure 3.

\begin{tabular}{|lccc}
\hline & $\begin{array}{c}\text { Magnitude } \\
\text { (waves) }\end{array}$ & $\begin{array}{c}\text { Temporal } \\
\text { dependence }\end{array}$ & $\begin{array}{c}\text { Spatial } \\
\text { scale }\end{array}$ \\
Optics figure errors & 2.5 & Static & $\mathrm{d} / 4$ \\
Small scale errors & $0.01-0.2$ & Static & $<\mathrm{d} / 4$ \\
Pump induced & $2.5-3$ & $50 \mathrm{msec}$ & $\mathrm{d} / 4$ \\
Thermal effects & $2.5-5$ & 4 hours & $\mathrm{d} / 3$ \\
Buoyancy ("turbulence") & $0.5-0.9$ & sseconds & $\mathrm{d} / 10$ \\
\hline
\end{tabular}

Notes: -Magnitude expressed as peak-to-valley wavefront aberation (waves at $1.05 \mathrm{~m}$ ). $-\mathrm{d}$ is beam size, nominally $34 \mathrm{~cm}$.

Figure 2. Factors affecting wavefront quality on Beamlet.

\section{Absolute wavefront \\ (waves @ $1.05 \mu$ )}

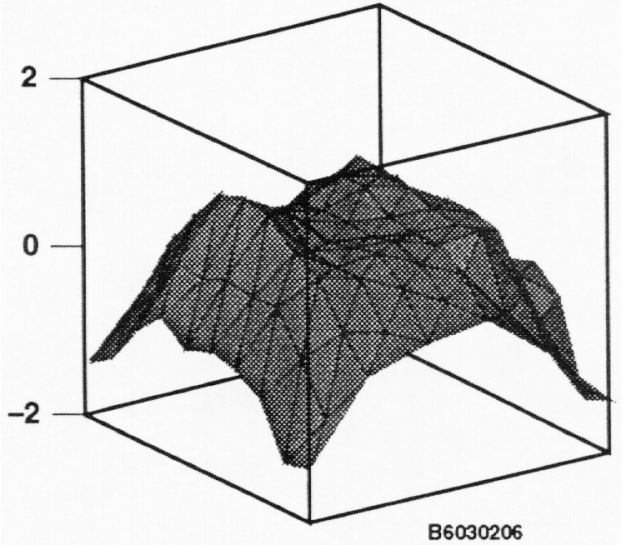

$p-v=2.47$ waves $r m s=0.62$ waves

\section{Static wavefront error} correction only

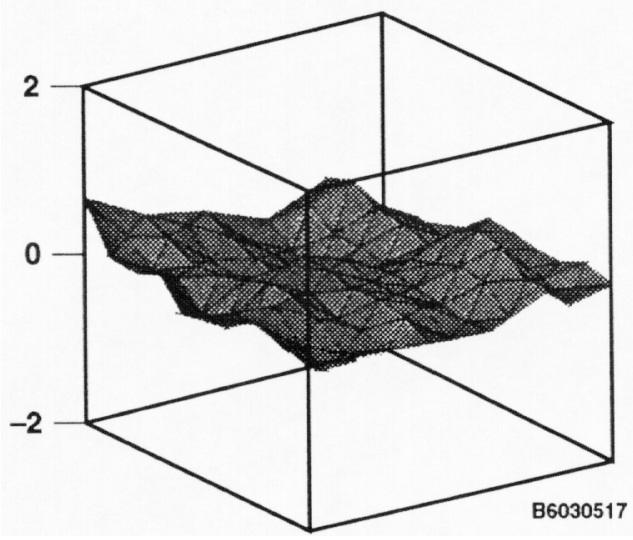

$p-v=1.18$ waves $r m s=0.19$ waves

With full prefigure: static and pump-induced wavefront error correction

70-50-1006-2450pto

Figure 3. Beamlet adaptive optics performance without the $t_{0}-1$ modifications. 
Initial operation of the Beamlet wavefront correction system employed manual reconfiguration for a shot: termination of closed-loop operation, removal of polarization rotators in the cavity spatial filter, exchange of wavefront sensor attenuators, and closure of a shutter on the cw laser. Figure 4 identifies the major components. These steps, which took about 10 minutes, provided for normal propagation of a pulse through the laser chain and prepared the wavefront sensor to record the pulsed wavefront. As suggested by Figure 1, however, useful correction for gas buoyancy effects requires that closed-loop operation be continued to within about 1 second of shot time and that reconfiguration occur automatically on a sub-second time scale.

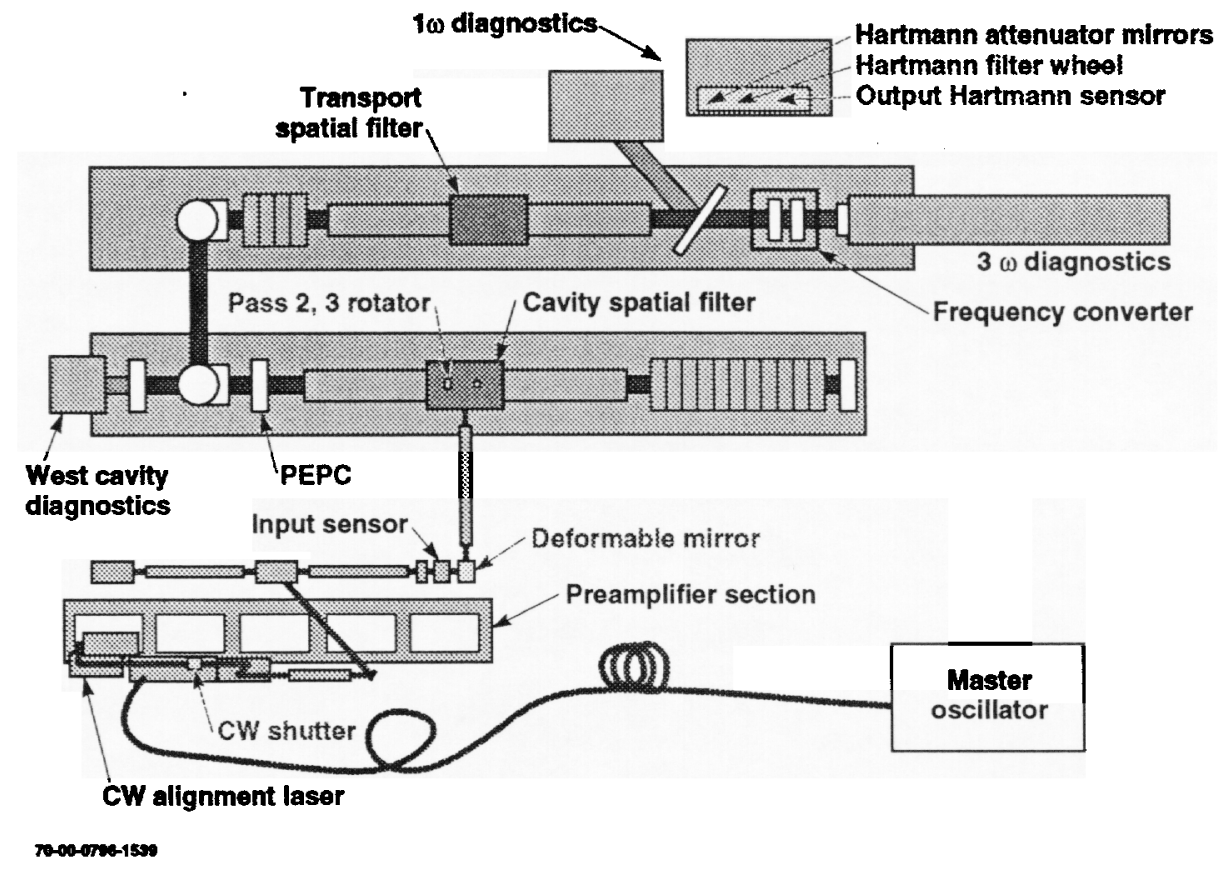

Figure 4. Major Beamlet subsystems and components modified for $t_{0}-1$ operation.

To accomplish these changes, we installed a fast shutter, a high speed filter wheel, and rapid-acting pneumatic translation stages for the polarization rotators and reflecting attenuator. These devices complete their activity within $500 \mathrm{ms,}$ and the closed-loop bandwidth of the modified wavefront correction system is $3 \mathrm{~Hz}$. This bandwidth, combined with the short time between opening the loop and firing a pulsed shot, is sufficient to significantly improve the wavefront quality of the pulse. The $\mathrm{cw}$ laser provides continuous illumination of the wavefront sensor as long as the wavefront loop is operating. Closing its shutter when the loop is opened prevents unwanted light into the camera at shot time.

\section{COMPONENTS}

There are several key components to the $\mathrm{t}_{0}-1$ wavefront control system. Continuous wave $\mathrm{cw}$ images are captured by a 77 element Hartmann-Shack sensor and a broadcast, video-type camera. Images are then digitized and processed by image processing hardware and software. The software compares images to an archived calibration image and calculates local slope errors based on image centroid analysis. Actuator stroke instructions are calculated by multiplying the slope erros by an inverted response matrix. The instructions are relayed to the deformable mirror through actuator drivers. In this manner, the wave front is corrected continuously.

In order to have closed-loop wavefront control up to one second before a system shot, we require a Timing Sequence Chassis. The Timing Sequence Chassis, as seen in Figure 5, monitors and controls the position of four components: the deformable mirror, the $\mathrm{cw}$ shutter, a fast, selectable neutral density filter wheel, the cw polarization rotators, and the antireflective/highly reflective filter combination to protect the Hartmann output sensor. The shutter used to tum off the cw light is a binary device. The filterwheel is a programmable, ten-position carousel, with filters graduated in half-step optical densities. Both it and the $\mathrm{cw}$ shutter are remotely operable, electrically driven devices. The polarization rotator assembly 
consists of two elements mounted to an air-driven servo positioner. The AR/HR device is also an air-driven device. The Timing Sequence Chassis also determines the mode of the deformable mirror control system.

The majority of our equipment "talks" via fiber, with appropriate twisted pair and coaxial cable connections to minimize capacitor bank discharge signal interference. Devices provide their "status" by an array of optical limiting switches providing logic to the DFM Timing Sequence Chassis. Much of the cabling to devices installed in vacuum spacial filters are shielded from UV radiation.

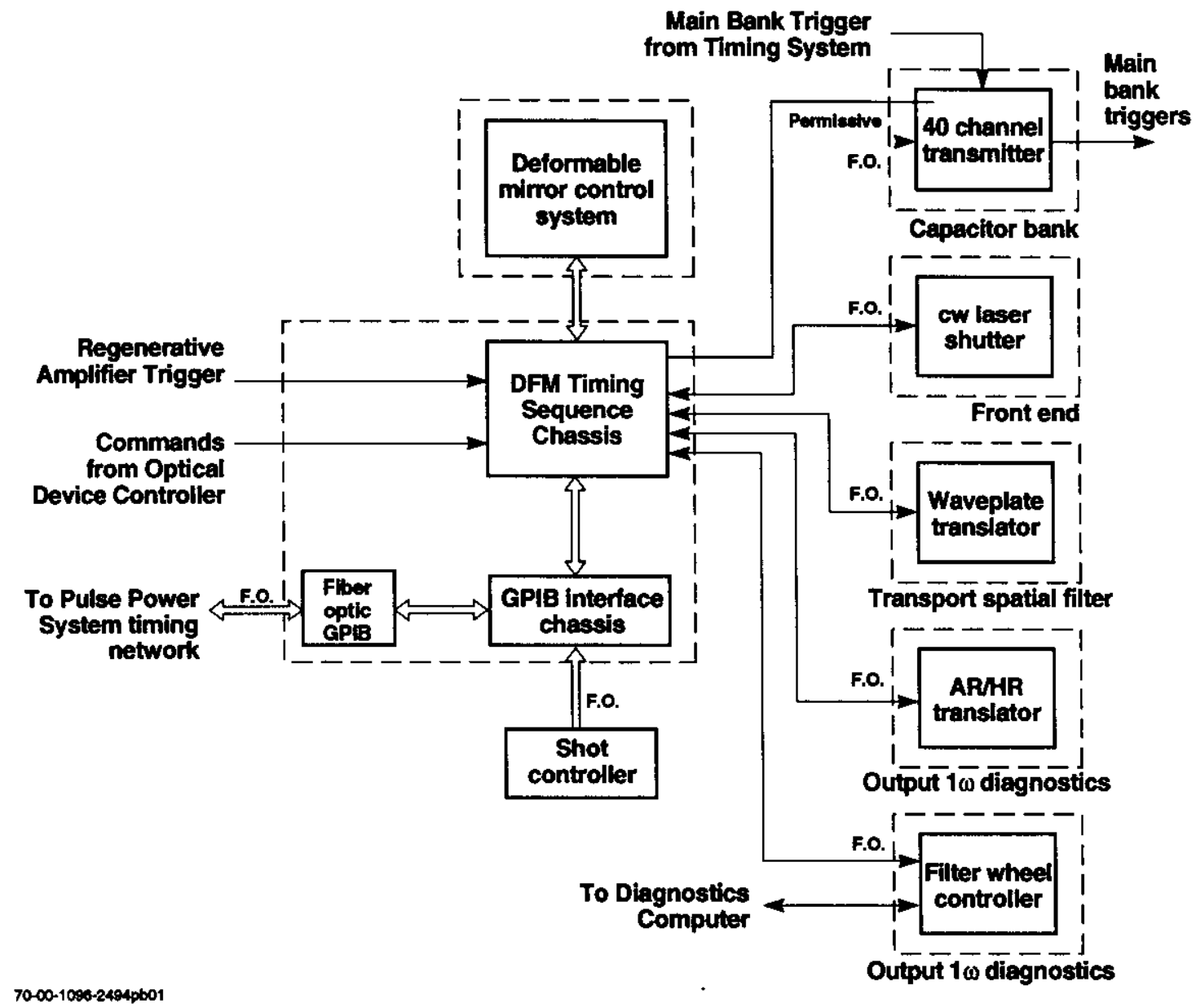

Figure 5. $t_{0}-1$ control block diagram.

\section{THE TIMING SYSTEM}

The $t_{0}-1$ DFM Timing Sequence Chassis in Figure 5 monitors the Pulse Power System Timing Network and the Regenerative Amplifier Trigger. The regenerative amplifier triggers every 5 seconds. The "shot controller" then sends a signal to the $t_{0}-1$ second DFM Timing Sequence Chassis to indicate that the next regenerative amplifier synchronization signal will trigger the capacitor bank. The DFM Timing Sequence Chassis waits 4 seconds, then prepares for the shot by initiating mode changes of the components it controls. After confirmation that the components have moved from their $\mathrm{cw}$ positions to their "system shot" positions, a "permissive signal" is sent to the capacitor bank allowing discharge for a system shot. This is usually accomplished within $\mathbf{5 0 0} \mathrm{ms}$. If a "permissive" is not sent to the capacitor bank, the shot is aborted and a report is sent to the shot controller to identify what optical component was improperly positioned. When the wavefront control system receives its signal from the timing system, it automatically unlocks the control loop and sets the Hartmann output camera to diagnose the shot wavefront. 


\section{5. to-1 MECHANICAL PERFORMANCE}

The mode transition time of $\mathrm{t}_{0}-1$ system components was measured. The air-actuated, mechanically dampened components moved in well under 1 second. Figure 6 indicates the extracton time of the polarization rotator, our slowest component. The $t_{0}-1$ gas pressure was set to 75 psi. Placing a photo-diode near the Hartmann output, we found that the full system transition time from closure of the cw shutter to flashlamp trigger was $955 \mathrm{~ms}$, as shown in Figure 7. Based on Figure 6, a shorter system transition time could probably be achieved.

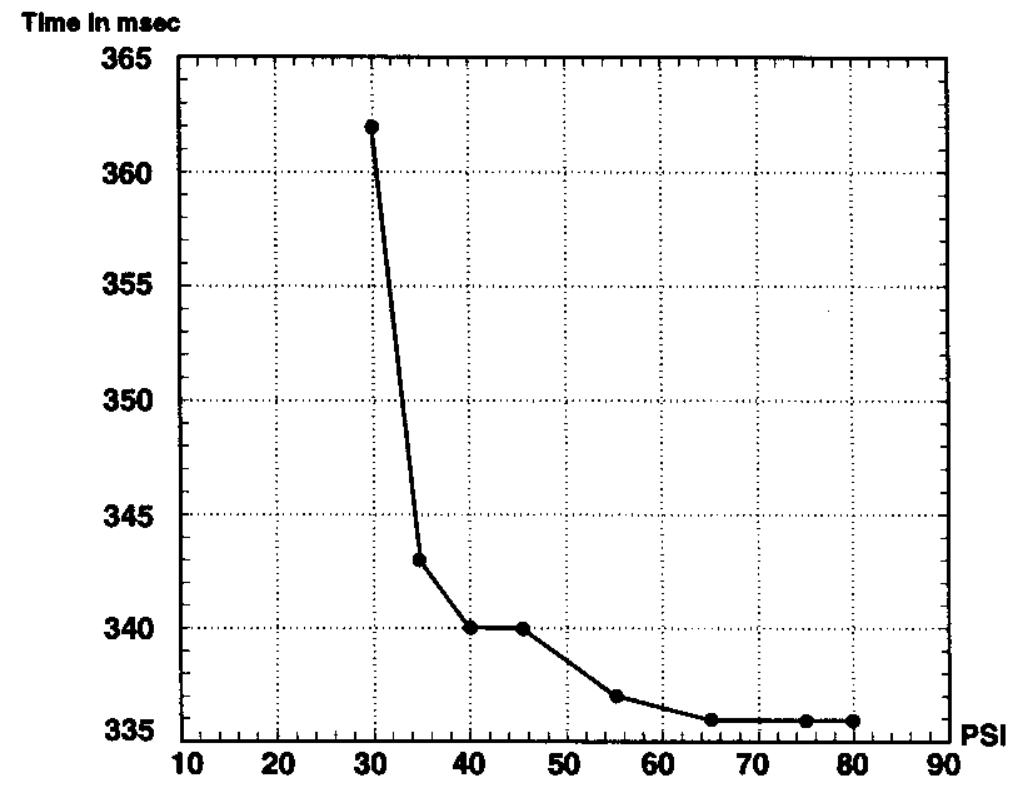

$70-50-1006-2451 p b 01$

Figure 6. Polarization rotator extraction time vs. gas pressure.

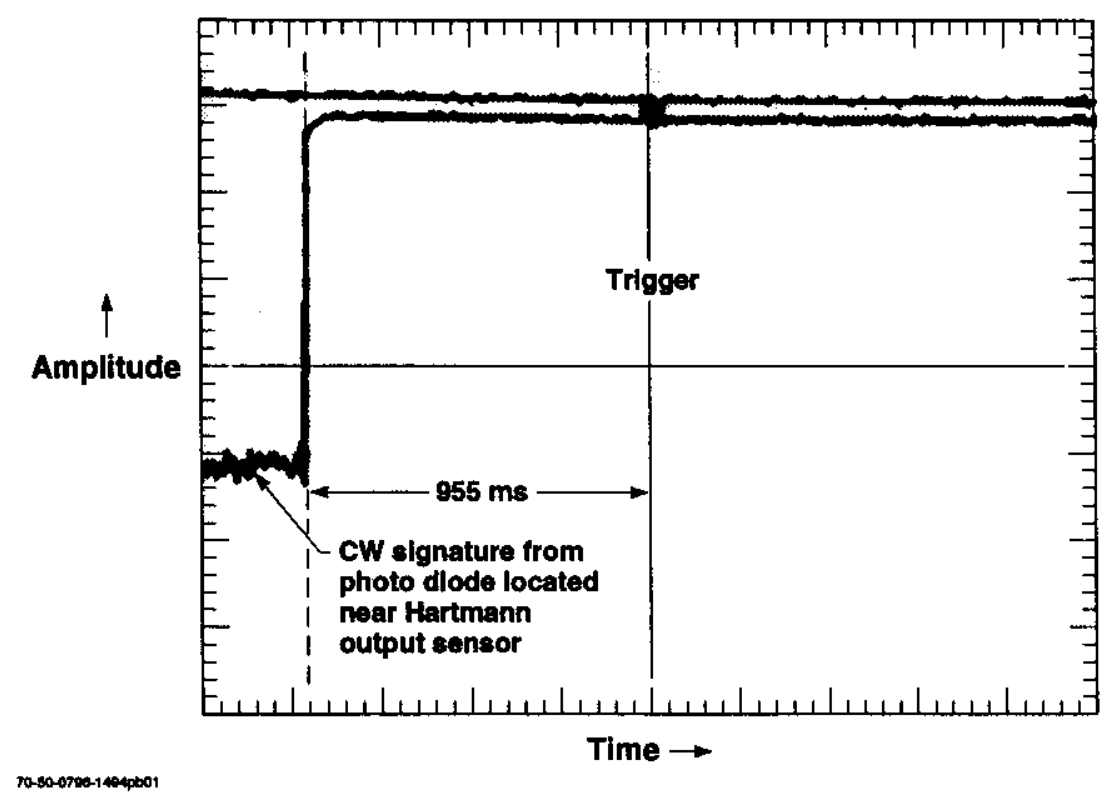

Figure 7. Shutter-closure to trigger elapsed time. 


\section{CONCLUSIONS}

We conducted a series of 22 shots to test the effectiveness of the $t_{0}-1$ wavefront control system. Pairs of rod shots were taken between flashlamp shots to provide thermal loading of the main cavity amplifiers. A rod shot is defined as a low energy shot where the main laser amplifiers are not fired. Each rod shot pair consisted of a shot with the $\mathrm{t}_{0}-1$ system engaged, and a shot without it engaged.

Referring to Figure 8, we see that the relative Strehl ratio or far-field brightness (normalized to first cold shot) is initially improved with the $t_{0}-1$ system in operation. However, after two flashlamp shots in two hours, the optics train is sufficiently saturated with thermal effects that there is no significant improvement in the wavefront from the $t_{0}-1$ system.

A plot of the measured azimuthal average intensity for a typical rod shot with the $t_{0}-1$ and without the $t_{0}-1$ system operating is shown in Figure 9. These shots were made about an hour and a half after the first flashlamp shot of the campaign. The peak intensity is about double and the intensity at angles from 4 to $8 \mu \mathrm{rad}$ is reduced in the shot with the active $t_{0}-1$ system compared to the shot without it.

More work is required to determine how much thermal loading the $t_{0}-1$ system can prefigure. The high-repetition shot sequence in this campaign intentionally denied the amplifier slabs the necessary cooling time. We believe that with a slower repetition rate and longer intervals between shots, as is planned for NIF, a $t_{0}-1$ system will consistantly improve the focus of the laser.

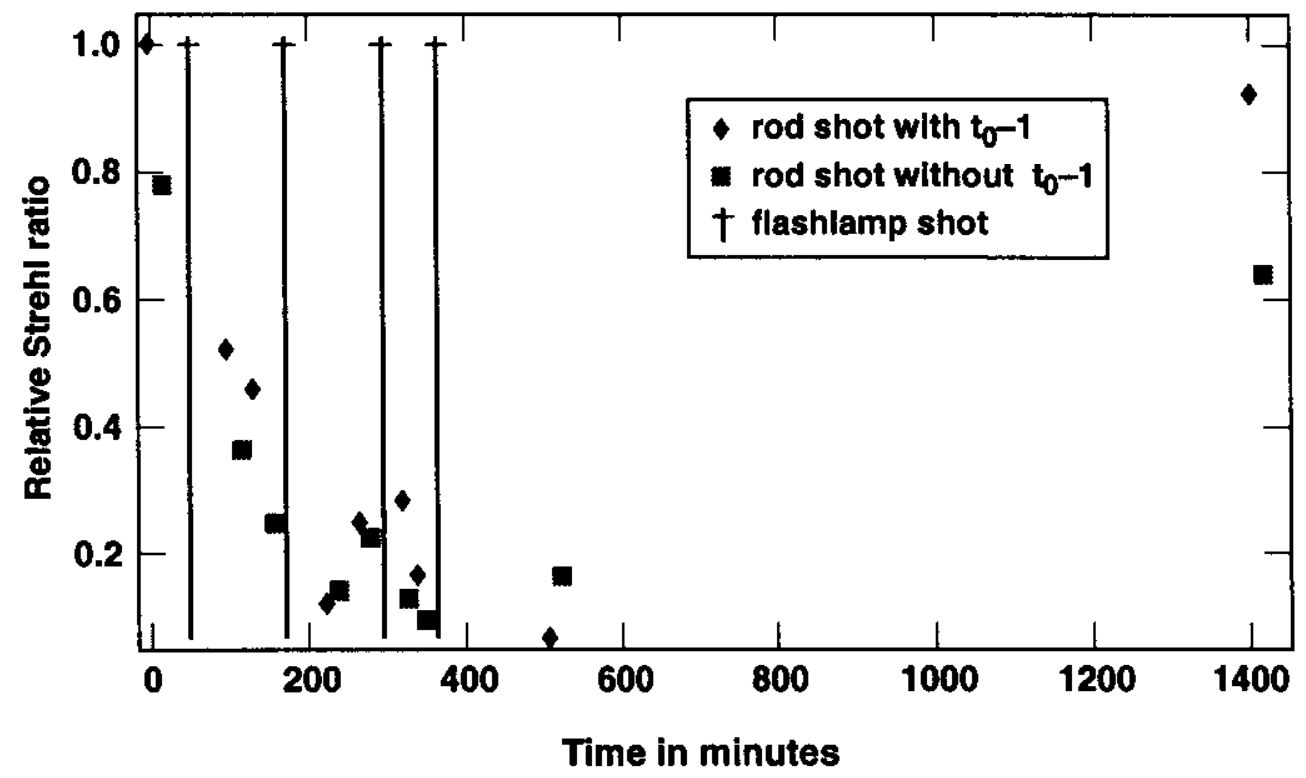

70-00-1006-2495bp01

Figure 8. Relative Strehl ratios vs. time with and without $\mathrm{t}_{0}-1$ system. 


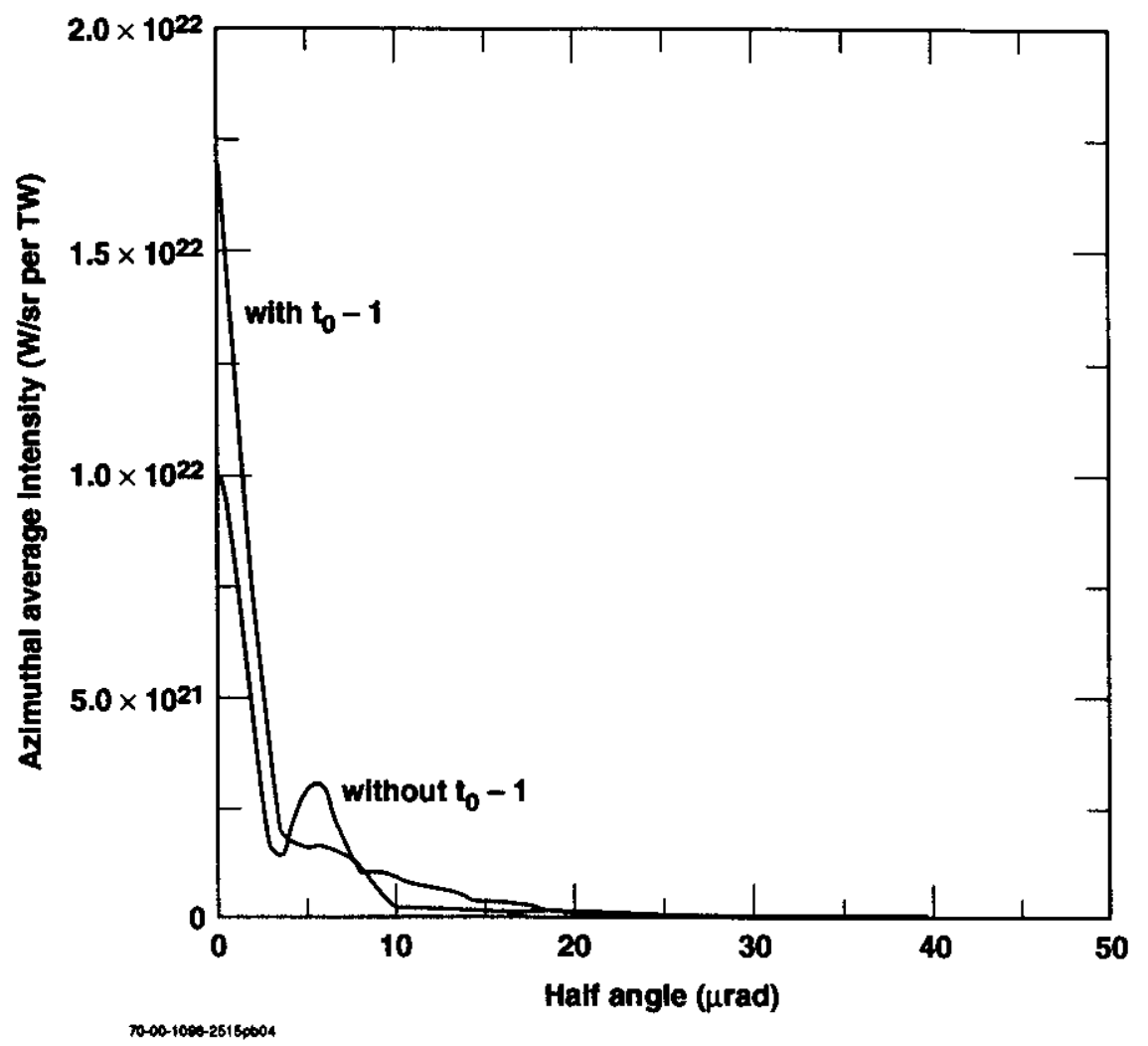

Figure 9. Azimuthal-average intensity with and without $t_{0}-1$ system.

\section{ACKNOWLEDGMENTS}

This work has been conducted for the ICF program and National Ignition Facility at Lawrence Livermore National Laboratory. The authors express their gratitude to the many individuals who contributed assistance and insight concerning this work.

Work performed under the auspices of the U.S. Department of Energy by the Lawrence Livermore National Laboratory under Contract W-7504-Eng-48.

\section{REFERENCES}

1. B.M. Van Wonterghem, J.T. Salmon, R.W. Wilcox, "Beamlet Pulse Generation and Wavefront Control System", Inertial Confinement Fusion Quarterly Report, Volume 5, Number 1, Oct-Dec 1994.

2. J.T Salmon, E.S. Bliss, T.W. Long, et al., "Active and Adaptive Optical Systems" (SPIE-International Society for Optical Engineering, Bellingham, WA, 1991: Proc. SPIE 1452) pp. 459-467. 


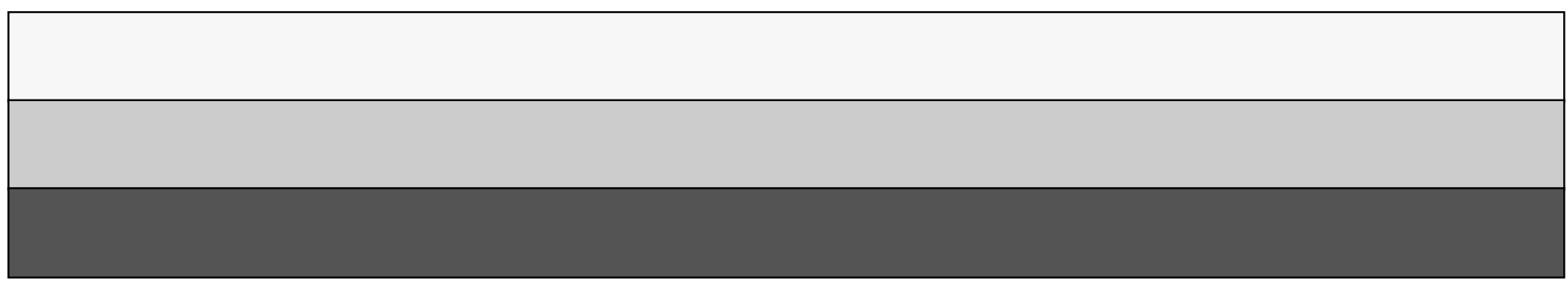

